\title{
Search for neutrino radiative decays during total solar eclipse
}

\author{
Vlad Popa*† \\ INFN, I-40129 Bologna, Italy and ISS, R-77125 Bucharest, Romania \\ E-mail: popa@bo.infn.iti
}

ABstract: We present the results of the measurements performed in the occasion of the 2001 total solar eclipse, looking for visible photons emitted through a possible radiative decay of solar neutrinos. We establish lower limits for the $\nu_{2}$ and $\nu_{3}$ proper lifetimes above $10^{3} \mathrm{~s} / \mathrm{eV}$, for neutrino masses larger than $10^{-2} \mathrm{eV}$.

\section{Introduction}

The data accumulated in the last few years in favor of neutrino oscillations (both solar,

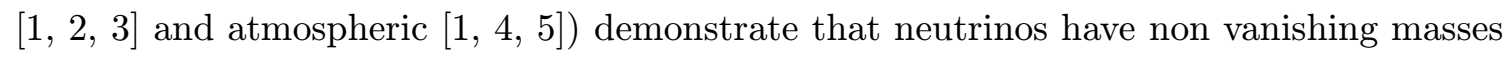
(for recent reviews see $\left.\left[\bar{b}_{1}^{1}, \underline{\underline{Z}}_{1}\right]\right)$. The most common interpretation of the oscillation data is based on two-generation mixing scenarios; the solar $\nu_{e}$ neutrinos are supposed to be a mixing of two mass eigenstates, $\nu_{1}$ and $\nu_{2}$, with $m_{2}>m_{1}$ (in the "normal hierarchy"):

$$
\left|\nu_{e}\right\rangle=\left|\nu_{1}\right\rangle \cos \theta_{12}+\left|\nu_{2}\right\rangle \sin \theta_{12}
$$

where $\theta_{12}$ is the mixing angle. A more complete description would require the consideration of three neutrino generation mixing, but the available data do not allow to determine all the corresponding mixing parameters.

If the neutrino mass states have also a non-vanishing magnetic moments, radiative decays

$$
\nu_{i} \rightarrow \nu_{j}+\gamma
$$

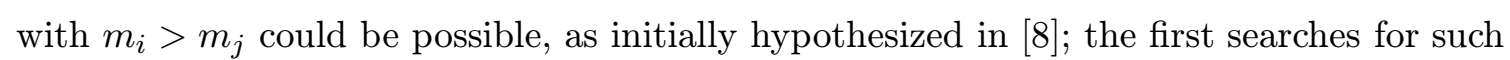
decays were based on astrophysical considerations (see eg. $\left[\underline{9}\left[\begin{array}{l}9 \\ 0\end{array}\right]\right)$. The status of the decaying theory and phenomenology was summarized in [i] $\left.{ }_{1}^{1} \overline{0}\right]$.

\footnotetext{
${ }^{*}$ Speaker.

${ }^{\dagger}$ for the NOTTE Collaboration: S. Cecchini, D. Centomo, G. Giacomelli, R. Giacomelli, V. Popa, C.G. Şerbănuţ and R. Serra
} 
The astrophysical neutrino lifetime lower limits are usually large (e.g. $\tau_{0} / m>2.8 \times 10^{15}$ $\mathrm{s} \mathrm{eV}^{-1}$ where $\tau_{0}$ is the lower proper lifetime limit for a neutrino of mass $m$, [i] $\left.\mathrm{i}_{1}^{1} \mathrm{l}_{1}\right)$, but they are indirect and rather speculative limits.

Much lower "semi-indirect" limits were deduced from the re-interpretation of solar and atmospheric neutrino data. Earlier attempts to explain the solar neutrino or atmospheric neutrino anomalies only in terms of neutrino decay have been ruled out by the existing evidence [1] $\left.{ }_{1}^{1} \overline{2}\right] ;$ the present accepted explanations are based on neutrino oscillations, but do not exclude the hypothesis of neutrino decays. As an example, from the SNO data [2, 20 , proper lower limit of $\tau_{0} / m>8.7 \times 10^{-5} \mathrm{~s} \mathrm{eV}^{-1}$ was deduced [i] $\overline{3}$ i]. By analyzing all available solar neutrino data, stronger limits were obtained [i] $\left.{ }_{1}^{1} \overline{4}_{-1}^{1}\right]: \tau_{0} / m>22.7 \mathrm{~s} \mathrm{eV}^{-1}$ for the MSW solution, and $\tau_{0} / m>27.8 \mathrm{~s} \mathrm{eV}^{-1}$ for the vacuum oscillation solution of the solar neutrino problem (SNP).

Direct searches for radiative neutrino decays have been also performed. As an example we quote here the search for decay photons in the visible spectrum performed in the vicinity of a nuclear reactor [i] $\left.{ }_{1}^{1} \overline{1}\right]$, yielding $\tau_{0} / m$ lower limits in the range $10^{-8}$ to nearly $0.1 \mathrm{~s} \mathrm{eV}^{-1}$, assuming neutrino relative mass differences $\Delta m / m$ between $10^{-7}$ and 0.1 . Recently, a search for $\gamma$ photons, using the Prototype Borexino Detector at Gran Sasso [1] $\underline{1}_{-1} \bar{n}$ ] reported $\tau_{0} / m$ lower limits of $1.5 \times 10^{3} \mathrm{~s} \mathrm{eV}^{-1}$ (assuming a polarization parameter $\alpha=-1$ for the parent neutrino), $4.4 \times 10^{3} \mathrm{~s} \mathrm{eV}^{-1}$ (for $\alpha=0$ ) and $9.7 \times 10^{3} \mathrm{~s} \mathrm{eV}^{-1}($ for $\alpha=+1$ ).

Total solar eclipses (TSO) represent a particular opportunity to look for radiative decays of solar neutrinos in the visible spectrum, during their flight from the Moon to the Earth, inside the shadow cone produced by the Moon. The first experiment based on this idea was performed in October 24, 1995 [1] $1 \overline{1}$, and a lower limit for the $\nu_{2}$ proper lifetime $\tau_{0}$ of about $10^{2}$ s was obtained, assuming neutrino masses of few $\mathrm{eV}$ and $\Delta m_{21}^{2}=m_{2}^{2}-m_{1}^{2} \simeq 10^{-5}$ $\mathrm{eV}^{2}$.

Some of us intended to perform measurements along this line during the 1999 TSE, in Romania: two experiments were prepared, one airborne and one at mountain altitude, but the weather conditions made the observations impossible [1] $\left.{ }_{1}^{1} \overline{1}\right]$. We could only Analise a video film recorded by a local television, obtaining $\nu_{2}$ lower lifetime limits $1.8 \times 10^{-2}<$

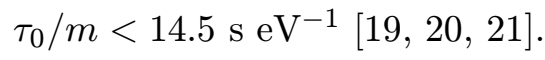

In this paper we present the results obtained from the analysis of our 2001 TSE observations.

\section{Experimental data}

The July 21, 2001 TSE was observed from a location near Lusaka (Zambia) (1456' lat. S, $28^{\circ} 14^{\prime}$ long. E, and $1200 \mathrm{~m}$ a.s.l.), at about $8 \mathrm{~km}$ from the line of centrality. We used two instruments: a digital videocamera with an optical zoom $10 \times$ and an additional $2 \times$ lens (which we will refer in the folowing as device " $\mathrm{A}$ ") and a small Matsukov - Cassegrain telescope ( $\phi=90 \mathrm{~mm}, f=1250 \mathrm{~mm}$ ) coupled to a digital camera (device "B"). The fields covered by a single pixel where about 10 " $\times 10$ " for images A and about 1.14 " $\times 1.14$ " for B. The experimental data consist in 4149 frames obtained with the videocamera (data set "A") and 10 digital pictures obtained with the telescope (data set "B"). Fig. II' shows two 


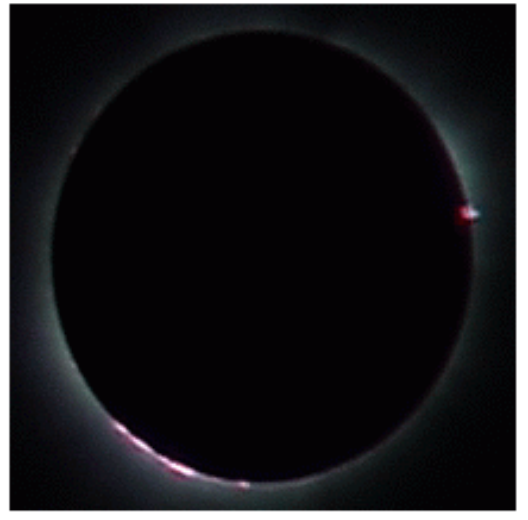

(a)

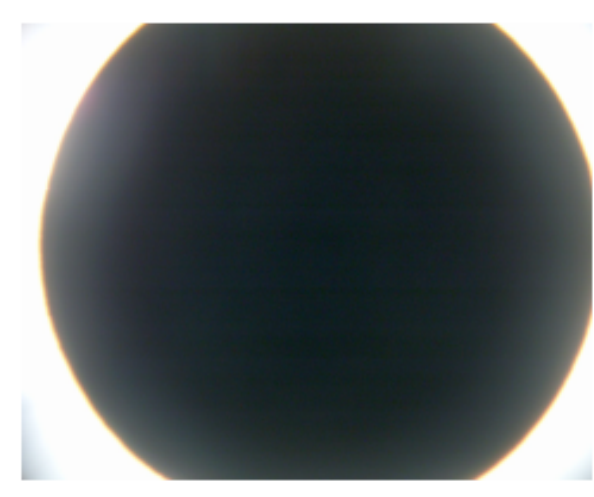

(b)

Figure 1: Two sample images of the 2001 TSE extracted from (a) data set A, and (b) data set B. Note that the pictures are not at the same scale.

of those images. For our analysis we summed the three color channels (Red, Green and Blue) of the images, thus recomposing the "white" light signal. The totality phase of the TSE in our observation site was about 3.5 minutes, so the displacement of the Sun behind the Moon is not negligible. As the signal of solar neutrino decays should be correlated with the direction to the center of the Sun, we calculated, for each frame in both data sets, the relative position of the Sun and, after determining by fit the center of the Moon disk, we computed the shift to be considered when summing the images in order to have the center of the Sun in the same pixel.

Both instruments were calibrated at the Catania and Bologna Astronomical Observatories. In the exposure conditions of the eclipse, the number of visible photons required for producing $1 \mathrm{ADU}$ (Acquisition Digital Unit) was $7.310^{4}$ for instrument A and $8.910^{2}$ for device B.

\section{The simulation}

In order to extract physical information concerning a possible neutrino radiative decays from TSE data, a previous knowledge of the characteristics of the expected signal in mandatory. For the analysis of the 2001 data we developed a full 3-dimensional Monte Carlo (MC) simulation [22 2$]$, based on the predictions of a recent version of the Standard Solar Model

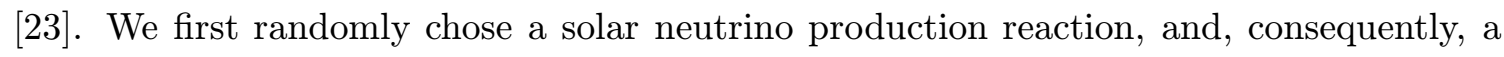
neutrino energy and the point of its creation inside the core of the Sun. Since we are interested only in neutrino decays that may produce signals in our detectors, we then generate a random photon arrival direction, inside the angular acceptance of our devices, and a decay point, uniformly distributed along the photon path, between the observation point (the Earth) and the Moon.

This procedure does not respect the kinematic probabilities of the simulated decay, so 
we attribute to each $\mathrm{MC}$ event a weight according the decay angular probability density:

$$
\frac{d \Gamma}{d \cos \theta^{*}} \propto \frac{m_{i}}{\left(\Delta m_{i j}^{2}\right)^{3}}\left(m_{i}^{2}+m_{j}^{2}+m_{i} m_{j}\right)\left(1+\alpha \cos \theta^{*}\right) .
$$

In Eq. Eq. 1.2.1. of the initial neutrino, in its center of mass reference frame. The polarization parameter $\alpha$ varies from -1 (left-handed) and 1 (right handed) for Dirac neutrinos, and is 0 for Majorana neutrinos. The kinematic weight for each MC event is obtained by integrating Eq. all photon directions that would lead to a signal in the same pixel, thus considering the experimental angular resolution. Note that such weights are dependent on the simulated device.

This MC incorporates both the realistic geometry of solar neutrino production and

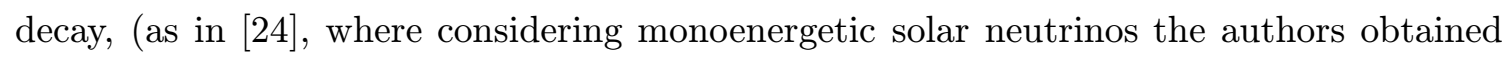
an analytical prediction) and the standard energy spectrum predicted by the SSM, (as in

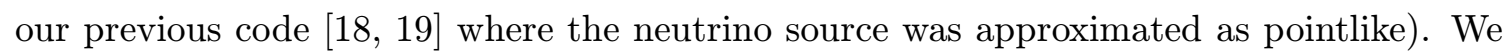
assumed that $m_{1}<m_{2}<m_{3}$ where $m_{1}, m_{2}, m_{3}$ are the masses of the $\nu_{1}, \nu_{2}$ and $\nu_{3}$ mass eigenstates, respectively. We restrict our analysis to a two generation mixing scenario, assuming the present mass differences obtained from solar neutrino experiments, the LMA solution with $\Delta m_{12}^{2}=6 \times 10^{-5} \mathrm{eV}^{2}$. Since SNO suggests also the presence of $\nu_{3}$ in the solar neutrino flux, we considered also the mass difference measured by atmospheric neutrino experiments: $\Delta m_{13}^{2} \simeq \Delta m_{23}^{2}=2.5 \times 10^{-3} \mathrm{eV}^{2}$.

Fig. 2 angular distance from the center of the Sun) from the simulation of (a) $\nu_{2} \rightarrow \nu_{1}+\gamma$ and (b) $\nu_{3} \rightarrow \nu_{1}+\gamma$ decays. The weights in Eq. $\overline{3}_{1} \overline{1}_{1}$ where calculated for different $m_{1}$ values. The histograms in Fig. concentrated at small $\theta_{E}$ angles (about $50 \mathrm{arcsec}$ ). The widths and shapes of the signals are sensitive to the mass assumed: the larger the mass, the narrower the signal band. In the case of Fig. $2 \bar{b}$, the signal is broader (about 250 arcsec) and is less sensitive to the mass choice.

One of the main results of the MC simulation consists in the determination of the global probabilities $P$ of a solar neutrino decay according to Eq. 1'.2, during its flight from the Moon to the Earth, and, produces a visible photon that reaches the detector. Those probabilities are both neutrino mass and instrument dependent. Consequently, assuming that an experiment detects $N_{\gamma}$ photons produced by neutrino radiative decays, the lifetime of the neutrino can be calculated from

$$
N_{\gamma}=P \Phi_{i} S_{M} t_{o b s}\left(1-e^{-\frac{\left\langle t_{M E}\right\rangle}{\tau}}\right) e^{-\frac{t_{S M}}{\tau}}
$$

where $P$ are the probabilities estimated by the MC simulation, $\Phi_{i}=\Phi_{\nu} \sin ^{2} \theta_{1 i},\left(\Phi_{\nu}\right.$ is the flux of solar neutrinos at the Earth (or Moon) and $\theta_{1 i}$ the mixing angle) is the local flux of solar $\nu_{i}$ mass eigenstate neutrinos, $S_{M}$ is the area of the Moon surface covered by the analysis and $t_{o b s}$ is the time of observation. $\left\langle t_{M E}\right\rangle$ is the average time spent by solar 


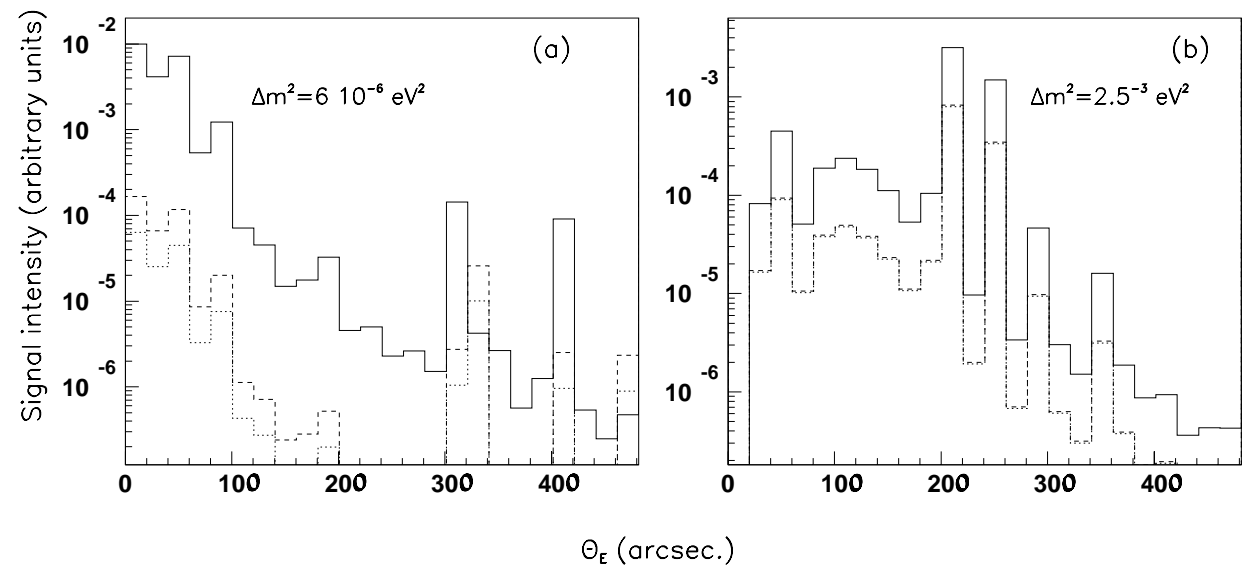

Figure 2: The expected shapes of the visible signals produced by the hypothesized solar neutrino radiative decay, assuming $m_{1}=0.001 \mathrm{eV}$ (solid histograms), $0.01 \mathrm{eV}$ (dashed) histograms) and $0.1 \mathrm{eV}$ (dotted histograms). The squared mass difference is assumed to be $6 \times 10^{-5} \mathrm{eV}^{2}$ (a) and $2.5 \times 10^{-3} \mathrm{eV}^{2}$ (b). In all cases $\alpha=-1$.

neutrinos inside the observation cone (one third of the flight time from the Moon to the Earth), and $t_{S M}$ is the time of flight of the neutrinos from the Sun to the Moon.

A complete discussion of the MC and of its results may be found in [22 2].

\section{Data analysis}

From the simulation described in the previous Section it follows that the expected visible signal from solar neutrino decays would have specific angular scales. A proper tool for investigating different scales of the eclipse images is the wavelet analysis. This technique gradually removes the contributions from various background sources as the diffraction of the coronal light on the borders of the Moon, the diffuse sky light, the ashen light (light reflected by the Earth on the surface of the Moon), etc. We used the simple Haar wavelet basis [i2 $2 \overline{5}]$. The $n$-order term of the decomposition is obtained by dividing the $N \times N$ pixels $^{2}$ image in square fields of $N / 2^{n} \times N / 2^{n}$ pixels $^{2}$ and averaging the luminosity in each field; the averages are then removed and the resulting image, the $n$-order residual, can be used to obtain the $(n+1)$-order term. Thus, each decomposition term results in an image in which objects of the corresponding scale are dominant, while the residuals contain information for smaller dimension scales.

As the wavelet analysis requires a dyadic dimension of the field (the number of pixels on each border of the image is a power of 2), we retained from our images $64 \times 64$ pixels (from data A) and $512 \times 512$ pixels (from data B) defined around the position of the center of the Sun, and summed them in order to increase the signal to background ratios. 


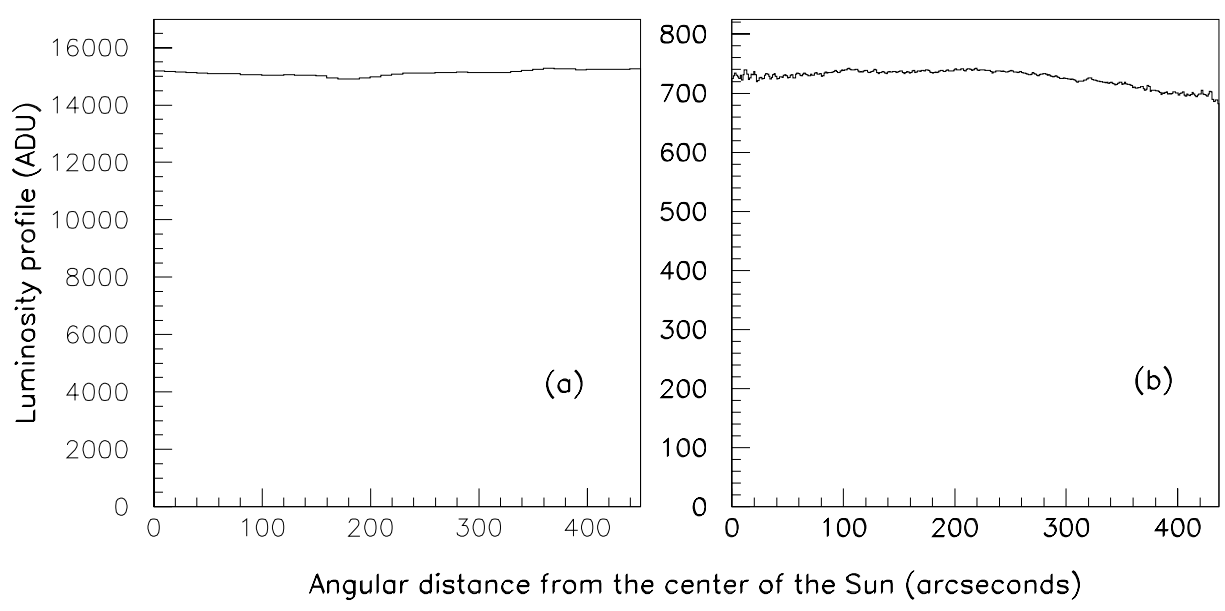

Figure 3: The luminosity profiles obtained from the raw data: a) data set A, b) data set B.

The decay signal is searched for by averaging the luminosity of the images over "rings" centered on the position of the center of the Sun. There is no "central pixel"; so we have considered each of the four pixels adjacent to the image center as "central" and then averaged the obtained luminosity profiles. Such profiles, obtained from the raw data (before the wavelet decomposition) are shown in Fig. '3-i. The difference between the two total images is due to the different CCD sensitivities, spatial resolution and optical features of the instruments. Data set A presents no clear structure, data B might contain some at relatively large $\theta$. In order to check that the shape of the luminosity curve in data set $\mathrm{B}$ could be produced by the ashen light, we aligned the 10 digital images along the direction of the center of the Moon, and made a similar analysis on a full Moon pictured obtained with the same instrument. Figs. 岱 show this comparison. In order to enhance the contrast of the images, from both pictures we removed the average luminosity, thus obtaining the $0^{\text {th }}$-order residual of the wavelet decomposition.

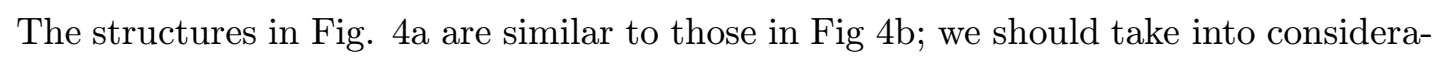
tion that the Earth reflects the light of the Sun as a convex mirror, thus the central part of the Moon receives more light from the Earth than the rest of it (the relative excess in the raw TSE data is only about $2 \%$ ). Instead the Sun illuminates the Moon uniformly. This observation suggests that we cannot simply remove the image of the full Moon from the data, as it would create a fake signal in the central part of the resulting image. Thus, one should develop a reliable model of the ashen light; alternatively one should use the wavelet decomposition. Note that the exposure conditions for the image of the full Moon were different than those during the eclipse, so the ADU values are not directly comparable. As we cannot determine which is the real contribution of the ashen light in set B, we can use the results only to determine a lower limit for the $\nu_{3}$ lifetime. 


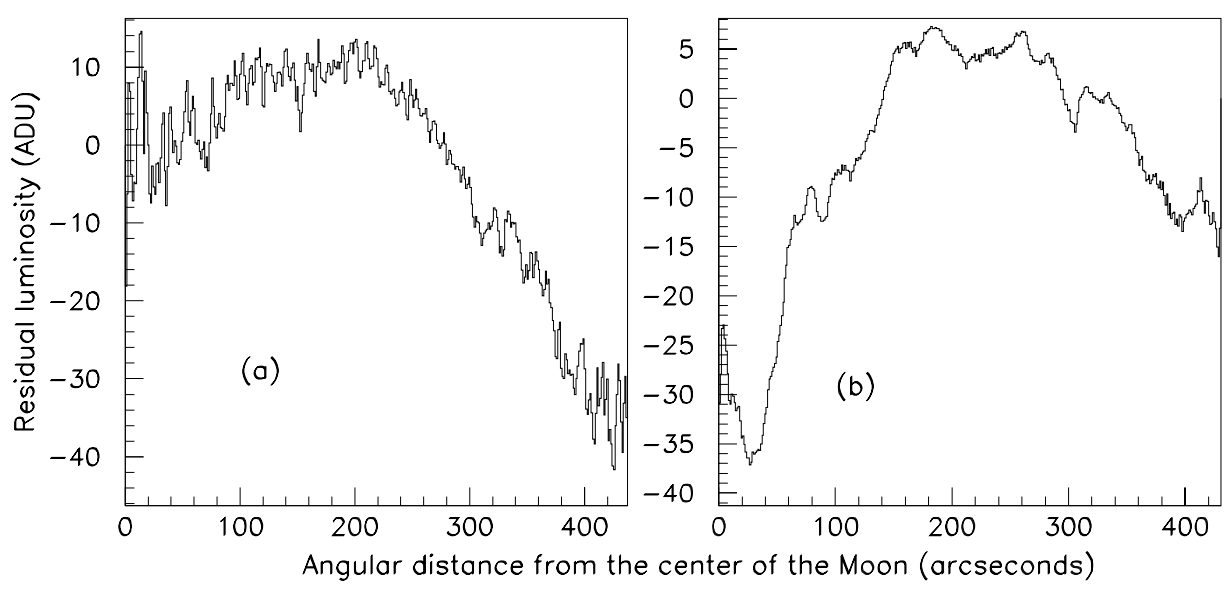

Figure 4: Light luminosity profiles after removing the average luminosity of (a) the sum of images in data set B aligned with respect to the center of the Moon, and (b): an image of the full Moon obtained with the same instrument.

(a)

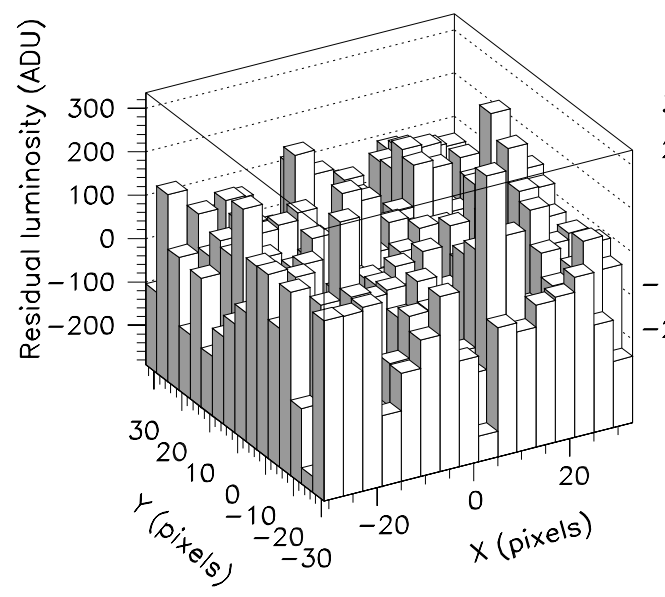

(b)

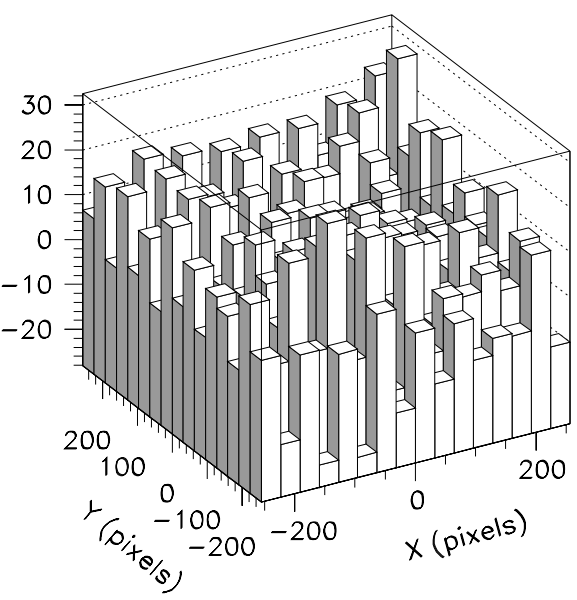

Figure 5: "White" light luminosity distributions of the fourth order wavelet term of (a) the summed images A and (b) B (centered on the Sun).

\subsection{Search for the $\nu_{2} \rightarrow \nu_{1}+\gamma$ signal}

The expected signal from a $\nu_{2} \rightarrow \nu_{1}+\gamma$ decay, considering its MC estimated width [2]2 $\left.2 \overline{2}\right]$, should be better seen in the fourth order wavelet term of data sets A and B, as it corresponds to structures with about 40" - 60" width. Figs. this term. Note that each bin is an average over $4 \times 4$ pixels in the case of data set A (Fig. 5a), and over $32 \times 32$ pixels for set B (Fig. 5b). No central maximum is present in both 


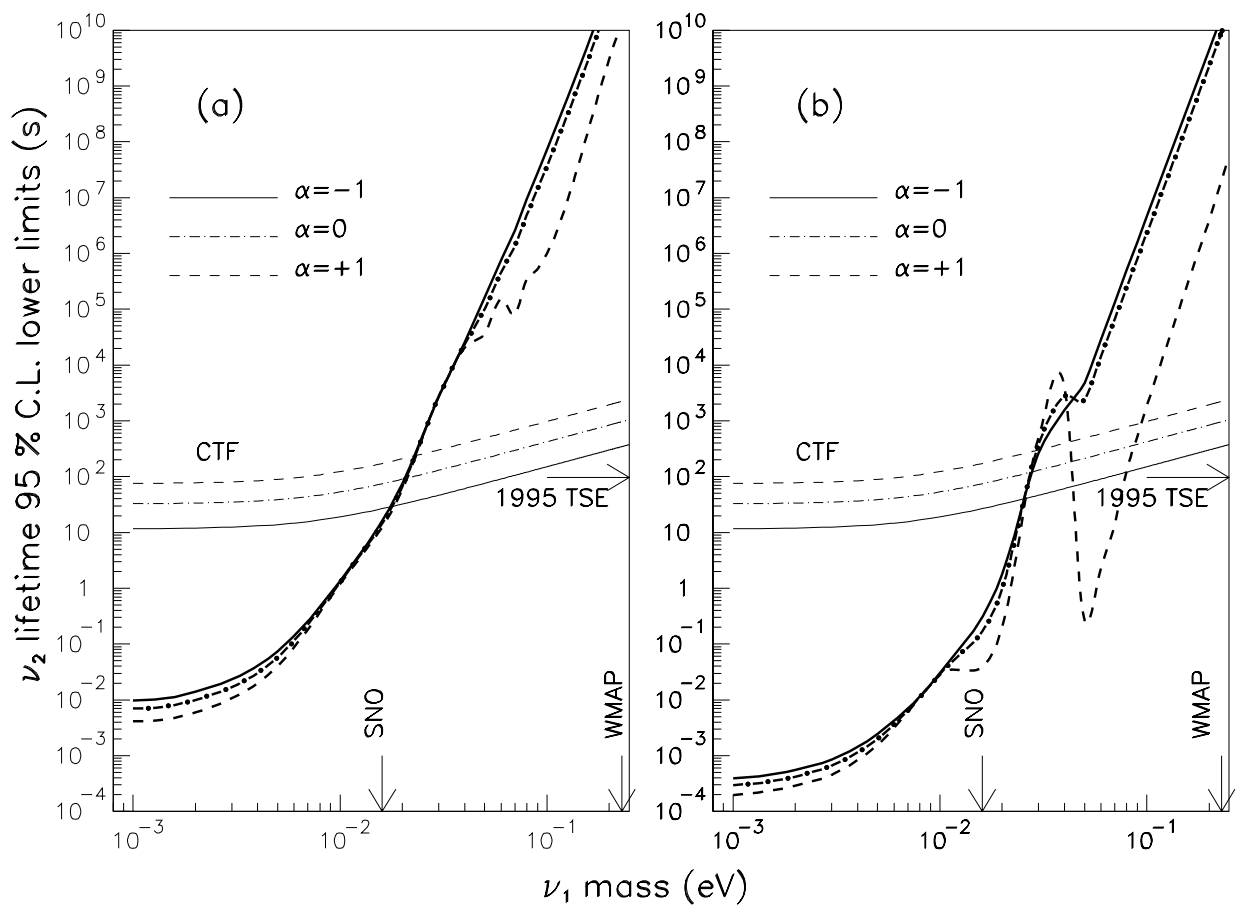

Figure 6: The 95\% CL lower limits for the $\nu_{2}$ proper lifetime, as function of the $m_{\nu_{1}}$, obtained from data sets (a) A and (b) B. The results are valid in a neutrino mixing scenario with only two generations, and $\Delta m_{2,1}^{2}=6 \times 10^{-5} \mathrm{eV}^{2}$. The discontinuities in the proper lifetime limits for righthanded neutrinos originate in the $\mathrm{MC}$ probabilities and reflect the changements in the initial neutrino energy imposed by the condition of obtaining visible decay photons pointing to the Earth. Other relevant limits are also indicated (see text).

data sets, and we can use these wavelet terms to determine lower lifetime limits for the investigated decay.

The $95 \%$ CL lower limits for the $\nu_{2}$ lifetime, in our case of no signal, are obtained by the substituting in Eq. $N_{\gamma}$ with $3 \sigma_{N_{\gamma}}$ of the forth order wavelet terms decomposition of the data, and considering $\sin ^{2} \theta_{i j}=\sin ^{2} \theta_{12} \simeq 0.74$ (the LMA solution of the "Solar Neutrino

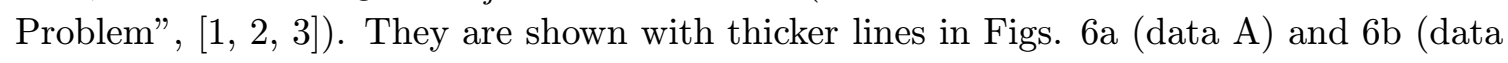
B), assuming that $\nu_{2}$ is a Dirac (lefthanded or righthanded) or a Majorana neutrino. The recent limits obtained from the Borexino Counting Test Facility [i] $\left.\overline{6}_{1}^{i}\right]$ are also shown, for comparison. The arrows labelled "SNO" and "WMAP" indicate the lower neutrino mass

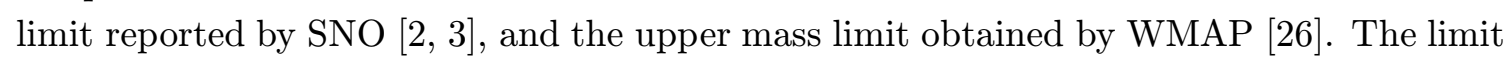
obtained by the first TSE experiment [i] in indicated by the horizontal arrow; note that this limit, obtained using different physical hypotheses, is valid for neutrino masses of few $\mathrm{eV}$.

Neutrino lifetime values larger than our lower limits are not in conflict with the oscillation explanation of the solar neutrino deficit. The neutrino time of flight from the Sun to the Earth is about $500 \mathrm{~s}$ (in the laboratory frame of reference). The Lorentz boost for 


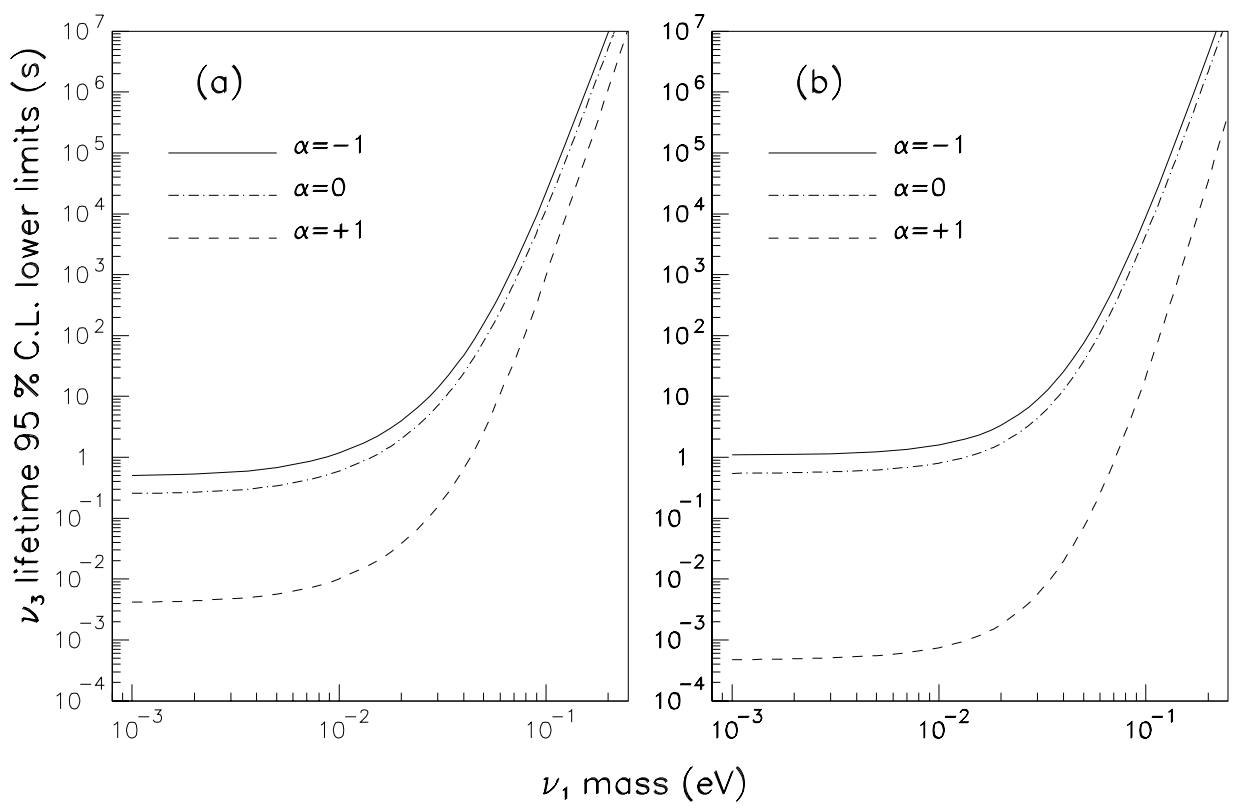

Figure 7: The 95\% CL lower limits for the $\nu_{3}$ proper lifetime, as function of the $\nu_{1}$ mass, obtained from (a) set A (a) and (b) B. The solid, dot-dashed and dashed lines correspond to three different neutrino polarizations, $\alpha=1,0$ and -1 , respectively. These results are obtained assuming $\Delta m_{3,(2,1)}^{2}=2.5 \times 10^{-3} \mathrm{eV}^{2}$ and $\sin ^{2} \theta_{13} \simeq 0.1$

a solar neutrino with a mass of about $0.02 \mathrm{eV}$ is $\gamma \simeq 1.5 \times 10^{7}$, so the fraction of $\nu_{2}$ that would decay into $\nu_{1}+\gamma$, assuming $\tau_{0} \simeq 60$ s (in the c.m.) would be only $\simeq 5 \times 10^{-7}$.

\subsection{Limits on the $\nu_{3} \rightarrow \nu_{1,2}+\gamma$ lifetimes}

As already shown, the search for $\nu_{3} \rightarrow \nu_{1,2}+\gamma$ signals is more difficult, as the ashen light could create a fake signature. Furthermore, the expected angular width of the expected signal is larger, so the wavelet decomposition could erase it. We still can compute 95\% CL lower limits for the corresponding lifetimes, substituting $N_{\gamma}$ in Eq. $\overrightarrow{3} \cdot \overrightarrow{2}$ with $3 \sigma_{N_{\gamma}}$ of the raw data $\mathrm{A}$ and $\mathrm{B}$. The mixing angle $\theta_{13}$ is not known, but it should be small; we assume $\sin ^{2} \theta_{13} \simeq 0.1$. The lifetime limits obtained in those conditions are shown in Fig. iti

\section{Conclusions}

We analyzed two sets of digital images obtained during the June $21^{\text {st }} 2001$ total solar eclipse, in Zambia, looking for possible radiative decays of solar neutrinos, yielding visible photons.

Data set A consists in a large number of frames recorded with a digital videocamera; it has a relatively large integration time, but a modest space resolution. 
Set $\mathrm{B}$ consists of 10 pictures taken with a digital camera coupled to a small telescope. Its time coverage is poorer than for set $\mathrm{A}$, but it has a better space resolution and the instrument sensitivity was an order of magnitude better.

The proper lower lifetime limits (95\% CL) obtained for the $\nu_{2} \rightarrow \nu_{1}+\gamma$ decays of lefthanded neutrinos range from $\tau_{0} / m_{2} \simeq 10 \mathrm{~s} \mathrm{eV}^{-1}$ to $\simeq 10^{9} \mathrm{~s} \mathrm{eV}^{-1}$, for $10^{-3} \mathrm{eV}<m_{\nu_{1}}<$ $0.1 \mathrm{eV}$, see Fig. '海: These limits are among the best obtained from direct measurements, demonstrating the potentiality of neutrino decay experiments during total solar eclipses (or possibly made in space, using the Earth as light absorber [2] $\left.\overline{2}_{i}^{1}\right]$ ). The lab. lifetime limits are about $10^{7}$ times larger, thus the fraction of neutrino decays from the Sun to the Earth would be negligible.

A similar analysis was made for a possible $\nu_{3} \rightarrow \nu_{2,1}+\gamma$ decay, assuming $\sin ^{2} \theta_{31} \simeq 0.1$ (the value of this mixing angle is not known). No signal compatible with a possible $\nu_{3} \rightarrow$ $\nu_{2,1}+\gamma$ is seen. The obtained $95 \%$ C.L. $\nu_{3}$ proper lifetime lower limits, for $m_{1} \geq 10^{-2} \mathrm{eV}$ and for $\alpha=-1,0$, are about two orders of magnitude lower than for the $\nu_{2}$, Fig. i i⿱i

New observations, in better technical conditions, during forthcoming TSE's should be considered.

An attempt along these lines was made during the December 2002 eclipse, but the weather conditions in South Africa did not allow any observation. We intended to use three portable telescopes, equipped with astronomy type CCD's. The sensitivity would have been about two orders of magnitude better than what reported in this paper.

\section{Acknowledgments}

We would like to acknowledge many colleagues for useful comments and discussions. We thank the people of the Catania and Bologna Astronomical Observatories for their assistance during calibrations. Warm thanks are due to the Kiboko Safari, Lilongwe, Malawi, for their assistance during the expedition in Zambia. This work was funded by NATO Grant PST.CLG.977691 and partially supported by the Italian Space Agency (ASI), INFN and the Romanian Space Agency (ROSA). V.P. thanks the organizers of the AHEP-2003 Workshop, Valencia, for their hospitality.

\section{References}

[1] Y. Fukuda et al., Phys. Rev. Lett. 81 (1998) 1562

[2] Q.R. Ahmad et al. (SNO Coll.), Phys. Rev. Lett. 87 (2001) 071301

[3] Q.R. Ahmad et al. (SNO Coll.), Phys. Rev. Lett. 89 (2002) 011301

[4] M. Ambrosio et al., Phys. Lett. B434 (1998) 451; B517 (2001) 59; B357 (1995) 481

[5] M. Ambrosio et al., Phys. Lett. B566 (2003) 35; B478 (2000) 5

[6] G. Giacomelli and M. Sioli, Astroparticle Physics, hep-ex/0211035

[7] S. Pakvasa and J.W.F. Valle, Neutrino Properties Before and After KamLAND, hep-ph/0301061

[8] A.L. Melott, D.W. Sciama, Phys.Rev.Lett. 46 (1981) 1369 
[9] H.L. Shipman and R. Cowsik, Ap. J. 247 (1981) L111

[10] D.W. Sciama, Nucl.Phys.Proc.Suppl. 38 (1995) 320

[11] S.A. Bludman, Phys. Rev. D45 (1992) 4720

[12] A. Acker and S. Pakvasa, Phys. Lett. B320 (1994) 320

[13] A. Bandyopadhayay, S. Choubey and S. Goswami, Phys. Lett. B555 (2003) 33

[14] A.S. Joshipura, E. Massó and S. Mohanty, Phys. Rev D66 (2002) 113008

[15] J. Bouchez et al., Phys. Lett. B207 (1988) 217.

[16] A.V. Derbin and O.Ju. Smirnov, JETP Letters 76 (2002) 483

[17] C. Birnbaun et al., Phys. Lett. B397 (1997) 143

[18] S. Cecchini et al., Astrophys. and Space Sci. 273 (2000) 35

[19] S. Cecchini et al., Limits on radiative decays of solar neutrinos from a measurement during a solar eclipse, hep-ex/0011048

[20] V. Popa et al., Astrophys. and Space Sci. 282 (2002) 235

[21] G. Giacomelli and V. Popa, in: M. Baldo Ceolin (Edt.), Neutrino Oscillations in Venice, Edizioni Papergraf (2001) 321; hep-ex/0110013

[22] S. Cecchini et al., Astropart. Phys. (2003) -in print-; hep-ph/0309107

[23] J.N. Bahcall, M.H. Pinsonneault and S. Basu, Ap. J. 555 (2001) 990

[24] J.-M. Frère and D. Monderen, Phys. Lett. B431 (1998) 368

[25] Y. Fujywara and J. Soda, Prog. Theor. Phys. 95 (1996) 1059

[26] D.N. Spergel at al., Ap.J. 148 (2003) 175 